\title{
Understanding the relationship between egg- and antigen-based diagnostics of Schistosoma mansoni infection pre- and post-treatment in Uganda
}

\author{
Joaquín M. Prada ${ }^{1,2^{*}}$, Panayiota Touloupou ${ }^{3}$, Moses Adriko ${ }^{4}$, Edridah M. Tukahebwa4,
} Poppy H. L. Lamberton ${ }^{5,6+}$ and T. Déirdre Hollingsworth ${ }^{1,7+}$

\begin{abstract}
Background: Schistosomiasis is a major socio-economic and public health problem in many sub-Saharan African countries. After large mass drug administration (MDA) campaigns, prevalence of infection rapidly returns to pretreatment levels. The traditional egg-based diagnostic for schistosome infections, Kato-Katz, is being substituted in many settings by circulating antigen recognition-based diagnostics, usually the point-of-care circulating cathodic antigen test (CCA). The relationship between these diagnostics is poorly understood, particularly after treatment in both drug-efficacy studies and routine monitoring.

Results: We created a model of schistosome infections to better understand and quantify the relationship between these two egg- and adult worm antigen-based diagnostics. We focused particularly on the interpretation of "trace" results after CCA testing. Our analyses suggest that CCA is generally a better predictor of prevalence, particularly after treatment, and that trace CCA results are typically associated with truly infected individuals.

Conclusions: Even though prevalence rises to pre-treatment levels only six months after MDAs, our model suggests that the average intensity of infection is much lower, and is probably in part due to a small burden of surviving juveniles from when the treatment occurred. This work helps to better understand CCA diagnostics and the interpretation of post-treatment prevalence estimations.
\end{abstract}

Keywords: Schistosomes, Kato-Katz, CCA, Mathematical models, Diagnostics, Trace readings

\section{Background}

Schistosomiasis is a major public health and socioeconomic problem in many sub-Saharan African countries, with estimates of 240 million people infected [1]. The current strategy to control morbidity is based on mass drug administrations (MDA) with the anthelmintic drug praziquantel. However, this does not prevent reinfection, which can be very rapid, with prevalence often reported to quickly return to pre-treatment levels [2-5].

\footnotetext{
*Correspondence: j.prada@surrey.ac.uk

Poppy H. L. Lamberton and T. Déirdre Hollingsworth are joint senior authors. ${ }^{\dagger}$ Equal contributors

${ }^{1}$ Department of Mathematics, University of Warwick, Coventry, UK

${ }^{2}$ School of Veterinary Medicine, Faculty of Health and Medical Sciences,

University of Surrey, Guildford, UK

Full list of author information is available at the end of the article
}

The main diagnostic tool currently used to evaluate Schistosoma mansoni prevalence and intensity of infection is the Kato-Katz thick smear technique (KK) [6], which measures the number of parasite eggs in one (or more) $41.7 \mathrm{mg}$ smears taken from a stool sample. KK has a very high specificity $(\sim 100 \%)$, and gives a quantitative measure of the level of infection, the egg count, which is positively correlated with disease burden. However, it has some disadvantages, including large day-to-day variation in egg outputs and readings [7-9] and low sensitivity at low levels of infection. This low sensitivity is due to fewer eggs being present at low intensities of infection and therefore less likely to be within the small sample of faeces [5, 10-13]. Eggs are still excreted after recent treatment, and are viable; 
with heavy excretions that can occur over a week posttreatment [5].

The "point-of-care" circulating cathodic antigen test (CCA) that measures schistosomal antigen in the urine is an alternative technique to evaluate presence of infection. These are regurgitated gut antigens from feeding worms, which might include juveniles. There are no soluble egg antigen (SEA) tests available to specifically measure egg presence. CCA therefore measures antigens in active worm infections that disappear from the body within 24 to $48 \mathrm{~h}$ of clearing the infection [14]. These assays are commercially available, and are now being used for countrywide mapping of the prevalence of schistosomiasis [15-17]. CCA has a higher sensitivity than KK, particularly at low intensity of infection, but there are some indications that specificity might not be $100 \%[5,13,18,19]$. One of the challenges of estimating specificity is the lack of an accurate "gold standard" against which CCA can be evaluated [19-21]. CCA possesses other limitations, such as how to interpret "trace" results, which is believed to represent a large proportion of true positives although again remains under debate and may vary across differing endemicity level areas, with urine concentration and between test readers [21, 22].

Despite several community- and national level-based studies, the relationship between $\mathrm{KK}$ and CCA diagnosis is still not well understood. Latent Markov models [23], latent class analyses [13, 24] and generalized linear mixed models [15] have been used to help elucidate this and address the issues associated with "trace" readings. However, these studies have focused on data pre-treatment and these relationships may become more complex and particularly important after recent treatment.

In this paper, we combined the information from $\mathrm{KK}$ and CCA pre-treatment, one month post- and six months post-treatment in a high endemic $S$. mansoni area, to create a mathematical model of the relationship between the two diagnostics at an individual level. We fitted this model using a Bayesian framework and estimated true underlying schistosome prevalence. We then used the model to simulate populations and sample KK and CCA diagnostics, with the aim of comparing the error in the estimation of prevalence using either CCA or KK (with different numbers of stool samples analysed).

\section{Methods}

Data

The dataset used in this manuscript was sourced from a longitudinal study in three primary schools in Mayuge District, Uganda, a highly endemic area on the shores of Lake Victoria [5]. The students, between the ages of 6 and 12 , were sampled at baseline (before treatment), one month after treatment and six months after treatment. At each sampling time, KK was performed in duplicate over three days as well as one CCA from a single urine sample. The number of individuals tested was 364, although there are missing data in some entries, with some individuals not having all six KK results, these can be inferred by the model (see below). More information on the sample collection and processing details is outlined by Lamberton et al. [5].

\section{Base model}

We created a mathematical model of S. mansoni infection in humans, where we assumed a gamma distribution of true intensity of infection across the infected population combined with a negative binomial distribution of error in these estimations at each time-point, in a similar fashion to the zero-inflated model developed in Atlija, Prada et al. [25], see Additional file 1 for a detailed model description. This framework allows discrimination between infected and uninfected individuals, and we can estimate infection status based on the information we have from each individual's diagnostics results. Individuals with undetectable levels of infection can potentially be assigned to either the infected or uninfected group. We can therefore define individuals' infectious status as

$$
\text { Status }_{i, t}= \begin{cases}0 & \text { uninfected or undetectable infection } \\ 1 & \text { infected }\end{cases}
$$

where Status $=0$ means that the individual is uninfected or with undetectable levels of infection, while Status $=1$ means that the individual is infected (at either detectable or undetectable levels), for each individual $i$ at time $t$. The true prevalence at each time-point $t$, is the proportion of individuals with Status $=1$, henceforth referred to as $P_{t}$.

Each infected individual has an intensity of infection, $\lambda_{i, t}$, drawn from a gamma distribution of intensity across the population, with shape, $s_{t}$, and rate, $r_{t}$, parameters being estimated in the model at each time-point $t$,

$$
\lambda_{i, t}=\operatorname{Gamma}\left(s_{t}, r_{t}\right)
$$

Kato-Katz, like any other method for obtaining faecal egg counts is susceptible to measurement error, particularly at low levels of infection. Unlike previous work where a Poisson distribution was used [25], we instead employed a negative binomial distribution, as the variance in the counts is higher than the mean [26, 27], with an over-dispersion parameter $\omega$. Moreover, uninfected individuals by definition do not have schistosomes, therefore cannot have KK counts different from zero; based on the individuals' status estimated above, the KK diagnostic for individual $i$, at time $t$, for the repeated count $c$ would be: 


$$
K K_{i, t, c}= \begin{cases}0 & \text { If Status } \mathrm{i}_{\mathrm{i}, \mathrm{t}}=0 \\ \operatorname{NegBinomial}\left(\lambda_{i, t}, \omega\right) & \text { If Status } \mathrm{i}, \mathrm{t}=1\end{cases}
$$

Similarly, the individual's status will determine possible CCA test outcomes and since the CCA test used in the field is a semi-quantitative diagnostic, with possible values of negative $(-)$, trace, positive $(+)$, double positive $(++)$ and triple positive $(+++)$, we converted them into $0,1,2,3$ and 4 , respectively. For infected individuals, we calculated $P_{i, t}^{C C A}$, which is a value between zero and one, that depends on the estimated intensity of infection $\lambda_{i, t}$, via a logistic function with model parameters $k_{1}$ and $k_{2}$ being estimated

$$
P_{i, t}^{C C A}=\frac{1}{1+e^{-k_{1}\left(\lambda_{i, t}-k_{2}\right)}}
$$

We then used the $P_{i, t}^{C C A}$ value to simulate the CCA diagnostic outcome with a binomial distribution of $n=4$, such that highly infected individuals are more likely to be diagnosed positive $[$ or $(++) /(+++)]$. On the other hand, uninfected individuals, if the specificity of the diagnostic was $100 \%$, should have negative CCA. Because the specificity is lower, we allowed for some uninfected individuals to have trace results in the diagnostic, with a probability $P_{T r}$; the CCA diagnostic would therefore be:

$$
K K_{i, t, c}=\left\{\begin{array}{lr}
\operatorname{Bernouilli}\left(P_{T r}\right) & \text { If Status } \\
\mathrm{i}, \mathrm{t} & =0 \\
\operatorname{Binomial}\left(n=4, p=P_{i, t}^{C C A}\right) \text { If Status }_{\mathrm{i}, \mathrm{t}}=1
\end{array}\right.
$$

This particular framework assumes that KK specificity is $100 \%$, and thus uninfected individuals must have zero eggs, as well as limiting the possible CCA results for uninfected individuals to either (-) or trace. The true prevalence estimate (i.e. proportion of infected individuals, $\left.P_{t}\right)$ can then be compared to the KK and CCA prevalence from the raw data.

The model was fitted to all three time-points concurrently. We forced the mean intensity of infection (mean of the gamma distribution) for the time-point one month after treatment $(t=2)$ to be lower than at baseline (before treatment, $t=1$ ) and at six months posttreatment $(t=3)$ such that

$$
\frac{s_{2}}{r_{2}}<\frac{s_{1}}{r_{1}}, \frac{s_{3}}{r_{3}}
$$

Another model assumption that we made was that the relation between infection level and the CCA test result is independent of the prevalence, this assumes that test interactions only occur within an individual at any given time, and thus we fitted one $k_{1}$ and $k_{2}$ parameter for all three time-points. We also assumed a single overdispersion parameter $\omega$ across all time-points.

The model was run in a Bayesian framework using a Gibbs sampling package in R [28] ("jags" [29] and "runjags" [30]), with two independent chains, a 'burn-in' period of 1000 iterations and 10,000 samples. Uninformative priors where used. Convergence was assessed by visual examination of the trace plots and the Gelman-Rubin statistic. The run includes all 364 individuals, with up to six KK samples and one CCA value each for every time-point, with the missing KK data inferred by the model. We assessed the goodness-of-fit of the model by comparing draws from the posterior distribution to the data, see Additional file 1.

\section{Sampling from the model fit}

After the model fitting, as described in the previous section, we obtained a posterior distribution for each of the fitted parameters (shapes and rates of the gamma distribution at each time point, $\omega, k_{1}$ and $k_{2}$ and $P_{T r}$ ). We explored the relationship between the estimated true prevalence and the average infection level, as prevalence on average decreases at lower infection levels. This relationship is not linear, and we sampled without extrapolating beyond the data, correlated values of average infection level and prevalence, see Additional file 2 . These values are used to generate simulated populations, and perform KK (two samples) and CCA tests on those populations. We compared how KK and CCA prevalence changes as true prevalence varies. We also explored the error in the estimation of prevalence using one to six $\mathrm{KK}$ and $\mathrm{CCA}$, assuming trace results as either positive $(\mathrm{CCA}+)$ or negative (CCA-).

\section{Results}

We compared prevalence estimations from the raw data (either KK or CCA) with our model estimation of true prevalence (Fig. 1). In general, both KK and CCA underestimate the prevalence of schistosomiasis in our population compared to the model. It is important to note that trace results in the CCA test can be either considered infected (Fig. 1; CCA+, green triangle) or uninfected (Fig. 1; CCA-, blue square). Using trace results in the CCA as positive improves the estimation of prevalence over trace results as negative for $S$. mansoni in this high endemicity community, and is a better estimator than $\mathrm{KK}$ at all time points. We estimated the proportion of truly uninfected individuals that have trace CCA results (rather than negative) to be around $15 \%$, albeit our credible interval ranges from 4 to $27 \%$.

The posterior draws from the model fitting were used to compare average estimated underlying intensity and prevalence of infection in this community (Fig. 2). At baseline before treatment, the estimated true prevalence 


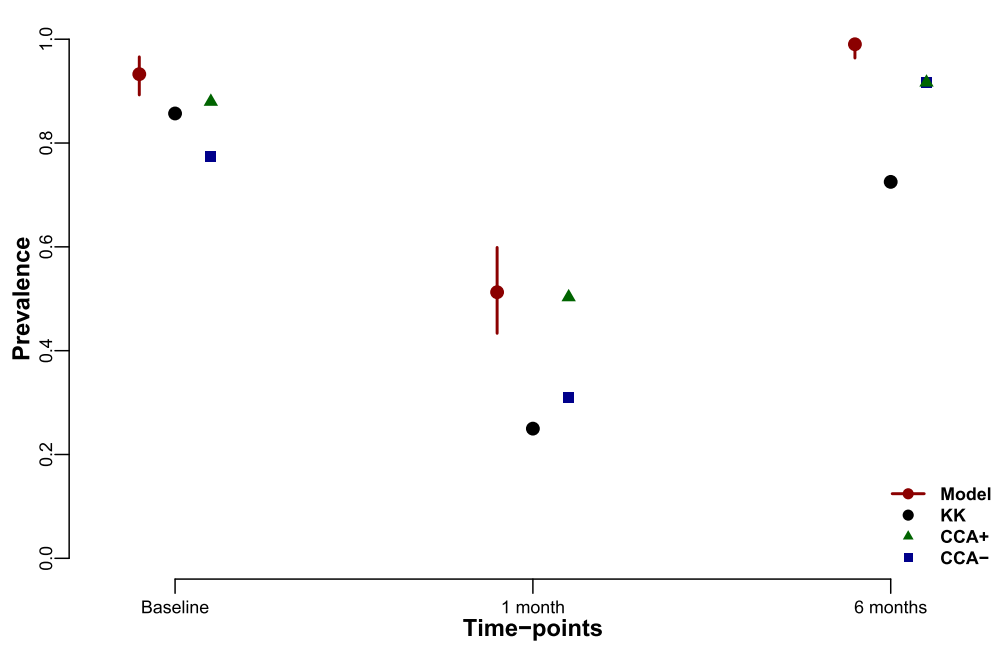

Fig. 1 Prevalence comparison between raw data and model prediction. Point estimates are the predicted values based on the raw data, black circles represent estimated prevalences using Kato-Katz, while the green triangles and blue squares are the estimates using a circulating cathodic antigen diagnostic, assuming trace results as positive (CCA+) and negative (CCA-), respectively. Red point with credible interval is the prevalence estimated by the model. Individual diagnostics prevalence estimates are generally lower than model predicted values combining both diagnostics

was high, as expected, and so was the average intensity of infection in the population (green area). One month after treatment, both the prevalence and average intensity of infection have drastically decreased (black area). However, six months after treatment (blue area), prevalence levels were similar to pre-treatment values, but the average intensity of infection was lower than at baseline, and moderately higher than one month after treatment.

We then interpolated across a range of infection levels to assess how the estimation of CCA and KK prevalence varies as true prevalence changes (Fig. 3a), and the relationship between both diagnostics (Fig. 3b). In Fig. 3a, the dotted diagonal indicates a diagnostic yielding the true prevalence, with points under the diagonal underestimating true prevalence, while the opposite is true for the points above the diagonal. As true prevalence increases, the difference in the prevalence estimation error between CCA and double KK diagnostics decreases. Only at high prevalence levels, from approximately over $70-80 \%$, we see KK (Fig. 3; black) giving in general higher prevalence estimates (and therefore closer to the true prevalence) than CCA assuming trace readings as negative (Fig. 3; CCA-, blue). Whereas, if we

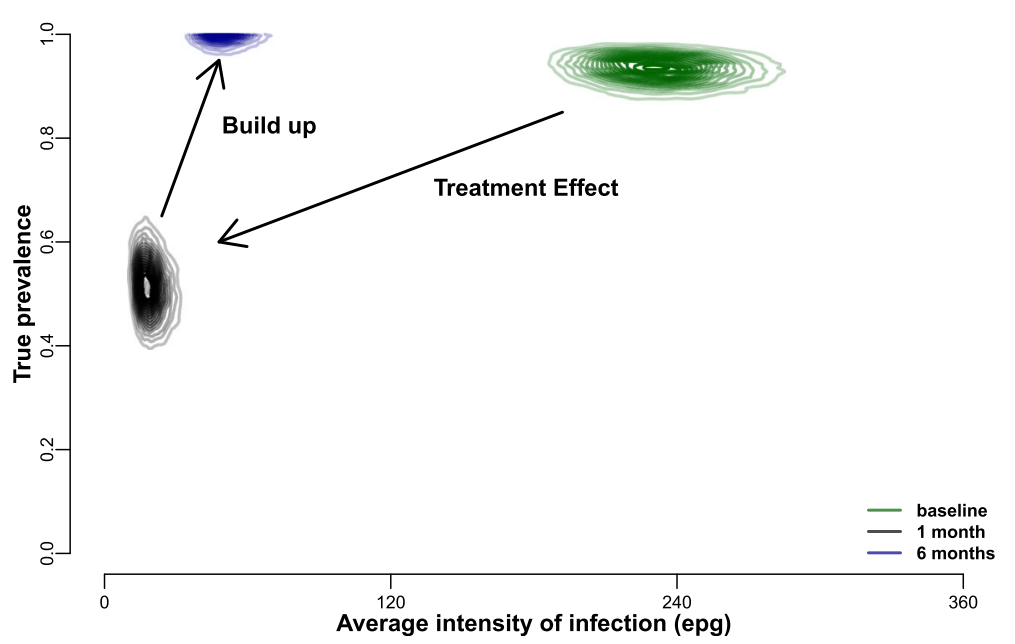

Fig. 2 Posterior distribution of average intensity of infection vs true prevalence. Each colour represent a different time point. At baseline (green) both prevalence and intensity of infection are high; one month post-treatment (black) both prevalence and infection are low; six months post-treatment (blue) the prevalence is similar to baseline, but the average population intensity of infection is still relatively low. This suggests that prevalence recovers faster in the population than intensity of infection, which builds up more slowly. The intensity of infection is shown in eggs per gram (epg) for clarity, however, the raw data are used in the model 

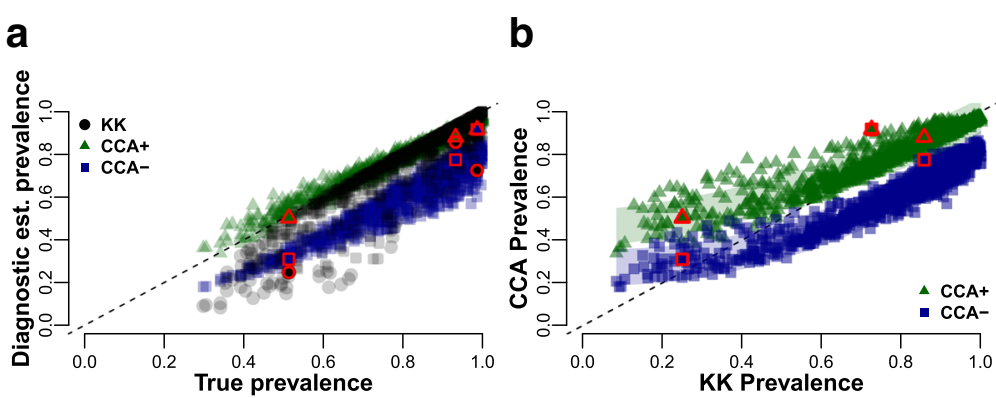

Fig. 3 Comparison between diagnostic estimates and true prevalence for simulated data. Colours represent the different diagnostics. Double KK estimates are coloured black (circles), CCA with trace results as positives (CCA+) are represented by green triangles, while CCA with trace as negative (CCA-) are blue squares. Symbols coloured red are the real data from the diagnostics for the average estimated true prevalence (Baseline and one and six months post-treatment). a Comparison of the different diagnostics and true prevalence illustrating that KK and CCA- always underestimate true prevalence, while CCA+ overestimates prevalence at low/medium prevalences. b Comparison of CCA diagnostics (CCA+ and CCA-) and KK diagnostics (two samples) showing a non-linear relationship as prevalence changes

consider a CCA trace reading as a positive result, CCA diagnostics would generally underestimate true prevalence above $85 \%$, but will otherwise tend to overestimate prevalence at lower levels of true prevalence (Fig. 3; CCA+, green). In Fig. $3 \mathrm{~b}$ we can see the non-linear relationship between both diagnostics. CCA+ diagnostics will rapidly overestimate prevalence compared to KK as prevalence decreases (i.e. above the diagonal). On the other hand, CCAestimates only give higher estimated prevalences than KK at low levels of prevalence, with both diagnostics underestimating true prevalence (Fig. 3). We did not simulate values below $\sim 35 \%$ true infection prevalence, as this was the lower value of estimated true prevalence in our data, corresponding to 1 month post-treatment (Fig. 3). It is important to note that prevalence estimated with either CCA- or KK always underestimated true prevalence in our simulations.

In the simulated population, we also mimicked different repeated KK test results, from one to six samples (informed by the data) and compared the error in the estimation of true prevalence (Fig. 4). The error in true prevalence estimation slightly decreased as more samples were examined by KK. However, CCA assuming trace results as positive (Fig. 4; green) had the smallest error in prevalence estimation, smaller than even six repeated KK. Of particular note is that using CCA but assuming trace readings were negative (Fig. 4; blue), had a significantly larger error than even a single KK across our simulated populations.

\section{Discussion}

We have performed an analysis to compare prevalence estimates using one to six repeated KK and a single CCA test, to understand how they differ from estimated true prevalence values. This is particularly important as current public health policy decisions are based on prevalence by KK [31], and the estimates obtained using CCA are often higher and will have a different degree of bias.
Moreover, since CCA is now being used in some countries to map schistosomiasis, it is crucial to understand how to interpret these new results so that control strategies can be updated accordingly and additional drug treatment requests and production be set in motion [22].

Kato-Katz smears have historically been vital for diagnosis and policy decisions and remain extremely useful at high levels of prevalence. This is because a high prevalence is associated with an average high intensity of infection. However, many highly endemic countries have been implementing mass control programmes for over a decade now and infection intensities in several regions, but not all, are now lower. Low sensitivity of the diagnostic test at low infection levels is now becoming an important issue. As shown in Fig. 1, the KK estimate of prevalence is significantly lower six months posttreatment than at baseline ( $10 \%$ lower estimate), even though the estimated true prevalence is the same. This

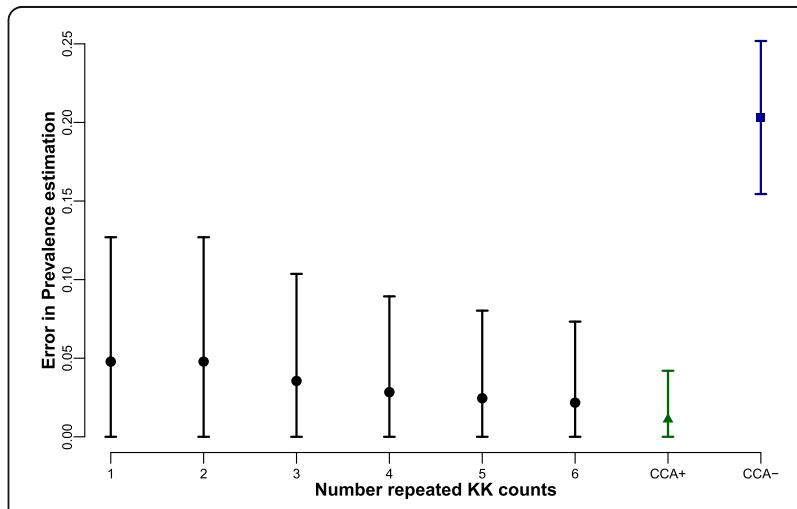

Fig. 4 Error in prevalence estimation based on simulated data. Mean and standard deviation across all simulated points. Error decreases as the number of repeated Kato-Katz measures increases but CCA assuming trace as positive (CCA+) has an overall smaller error in prevalence estimation. This suggests that multiple KK will improve precision, but not enough compared to CCA+, particularly at low levels of prevalence 
becomes apparent when looking at the average intensity of infection estimates which show much lower intensities six months post-treatment (Fig. 2). CCA, on the other hand, is generally a better estimator for true prevalence, particularly when assuming that trace results are positive, although this will lead to overestimation of prevalence at low true prevalences (Fig. 3a; green). This could be in part because even if a proportion of the trace results belong to uninfected individuals (we estimate it to be $15 \%$ on average), at a population level it helps minimize the underestimation of true prevalence inherent in the diagnostic (Fig. 4).

The relationship between prevalence estimates from $\mathrm{CCA}$ and $\mathrm{KK}$ as true prevalence changes is not linear (Fig. 3b) and in general, CCA is a better estimator of prevalence, particularly at low prevalences. The relationship we have estimated could be used to compare historical KK prevalence estimates to more contemporary, often higher CCA estimates. This is particularly helpful for programmes which have switched from KK to CCA and need to make informed public health decisions. However, it is still unclear exactly how the intensity of infection and prevalence increases after treatment over a short timescale, and more time-points are needed between one and six months posttreatment. In our approach we did not extrapolate beyond our data and therefore assumed the correlation between prevalence and intensity to be maintained.

It is also important to note that observing high prevalences (or even pre-treatment prevalence levels) a few months after treatment does not necessarily mean that intensities of infection are the same as before treatment (Fig. 2). With KK, we would potentially estimate a lower prevalence after treatment, because the average egg count in the population will be lower. However, using CCA as a diagnostic tool instead means that we would recover a better estimation of prevalence (i.e. a high prevalence), but we would miss the fact that the average intensity of infection is in fact reduced. A biological justification for this could be that praziquantel treatment has a higher efficacy in adult schistosomes and a reduced effect on juveniles [32, 33]. Therefore a few months after treatment, we would expect to see the juveniles becoming adults, but the overall infection load may still be low, due to a lower schistosome adult populations and only six months of new parasite exposure. This varying relationship between diagnostic test and accuracy in detecting prevalence versus intensity is important because public health guidelines for control are focused on prevalence data, whilst morbidity is positively correlated with infection intensity. Therefore, there is a potential disconnect between the prevalence indicators which $\mathrm{WHO}$ use for treatment guidelines and the intensity indicators which the WHO have as goals for morbidity control, with each of them also being affected by the choice of diagnostic test.
Future genetic studies may help elucidate the origin of the parasite eggs and/or antigens post-treatment, to decipher whether antigens are from new infections or surviving juveniles, which were not affected by the treatment, or whether they are from adult worms surviving the standard treatment and therefore resistant to the currently used drug praziquantel. In addition, the true meaning of a "trace" reading, and the absence of a "gold standard", may become more important after recent praziquantel treatment than our current model can explain. Correlations held at low intensities pre-treatment in low endemicity area, may not hold for similar low intensities (measured by KKs) post-treatment. These two situations, which may have similar mean eggs per gram values measured by KKs alone could reflect vastly different biological scenarios. Recent praziquantel treatment may interact with parasite juvenile and adult worm numbers, and egg production and clearance. All of these factors, plus the rate of continued parasite exposure and reinfection, strongly linked to endemicity and force of infection, may affect egg and/or antigen excretion at follow up. This in turn affects $\mathrm{KK}$ and/or CCA readings, blurring any pre-treatment correlations between KK and CCA outputs even further. This may in part explain why point estimates in Fig. 1 are more tightly clustered between diagnostic techniques than post-treatment.

\section{Conclusions}

We have shown the relationship between the two most commonly used diagnostic tools for S. mansoni infection, an egg-based and an antigen-based diagnostic. Having multiple diagnostics for a particular disease is becoming more common, but connecting the alternative diagnostics can prove challenging. In neglected tropical diseases, new studies are being done in this scope, such as the work by Irvine et al. [34] in lymphatic filariasis diagnostics. Understanding the interactions between the different diagnostic tools is important, particularly for diseases such as schistosomiasis that have ambitious targets for control and elimination by 2020. This study improves our understanding of these interactions and how to interpret prevalence and intensity measures of S. mansoni infection pre- and post-treatment.

\section{Additional files}

Additional file 1: Detailed model description. Document explaining and
justifying all model assumptions. Posterior distributions from the analysis
are also shown, as well as the fit to the data. (PDF $2476 \mathrm{~kb}$ )
Additional file 2: Sampling interpolation methodology. Methodology to
sample correlated infection levels and average intensity of infection. (PDF $82 \mathrm{~kb}$ )
Additional file 3: Dataset. (CSV $21 \mathrm{~kb})$

Abbreviations

CCA: Circulating cathodic antigen; CCA + : Trace as positive CCA; CCA-: Trace as negative CCA; KK: Kato-Katz; MDA: Mass drug administration 


\section{Acknowledgements}

We would like to thank Sake de Vlas and Luc Coffeng for helpful advice and comments on earlier versions of this work. The views, opinions, assumptions, or any other information set out in this report should not be attributed to the Bill \& Melinda Gates Foundation and the Task Force for Global Health or any person connected with them.

\section{Funding}

JMP, PT and TDH gratefully acknowledge funding of the NTD Modelling Consortium by the Bill \& Melinda Gates Foundation in partnership with the Task Force for Global Health. PHLL is currently funded by an European Research Council Starting Grant [680088 SCHISTO_PERSIST] and a Medical Research Council Global Challenges Research Fund Foundation Award [MR/P025447/1]. Data collection by PHLL, MA and EMT was funded by PHLL's Medical Research Council PhD studentship and the Schistosomiasis Control Initiative.

\section{Availability of data and materials}

Dataset supporting the conclusions of this article is included within the article as Additional file 3. KK is reported in measured eggs (up to six counts each time-point), CCA ranges from 0 to 4 [from (-) to (+++)]. t0 is baseline, $\mathrm{t} 1$ and $\mathrm{t} 2$ are one month and six months post-treatment respectively.

\section{Authors' contributions}

JMP, PHLL and TDH conceived the study. PHLL, MA and EMT collected the data. JMP developed the model and performed the analysis. PT supported development of the statistical methodology. JMP, PHLL and TDH drafted the manuscript. All authors critically reviewed, read and approved the final manuscript.

\section{Ethics approval and consent to participate}

This manuscript uses anonymized human data previously published (see Lamberton et al. 2014 [5]) originally collected with the approval of the Uganda National Council of Science and Technology (Memorandum of Understanding: sections $1.4,1.5,1.6)$ and the Imperial College Research Ethics Committee (EC NO: 03.36. R\&D No:03/SB/033E).

\section{Consent for publication}

Not applicable.

\section{Competing interests}

The authors declare that they have no competing interests.

\section{Publisher's Note}

Springer Nature remains neutral with regard to jurisdictional claims in published maps and institutional affiliations.

\section{Author details}

'Department of Mathematics, University of Warwick, Coventry, UK. ${ }^{2}$ School of Veterinary Medicine, Faculty of Health and Medical Sciences, University of Surrey, Guildford, UK. ${ }^{3}$ Department of Statistics, University of Warwick, Coventry, UK. ${ }^{4}$ Vector Control Division, Ministry of Health, Uganda, Kampala, Uganda. ${ }^{5}$ Institute of Biodiversity, Animal Health and Comparative Medicine, University of Glasgow, Glasgow, UK. 'Wellcome Centre for Molecular Parasitology, University of Glasgow, Glasgow, UK. ${ }^{7}$ Big Data Institute, University of Oxford, Oxford, UK.

Received: 4 August 2017 Accepted: 6 December 2017 Published online: 08 January 2018

\section{References}

1. Gray DJ, McManus DP, Li Y, Williams GM, Bergquist R, Ross AG. Schistosomiasis elimination: lessons from the past guide the future. Lancet Infect Dis. 2010;10(10):733-6.

2. Gryseels B, Nkulikyinka L. Two-year follow-up of Schistosoma mansoni infection and morbidity after treatment with different regimens of oxamniquine and praziquantel. Trans R Soc Trop Med Hyg. 1989;83(2):219-28.

3. Ahmed AM, El Tash LA, Mohamed EY, Adam I. High levels of Schistosoma mansoni infections among schoolchildren in central Sudan one year after treatment with praziquantel. J Helminthol. 2011;86:1-5.

4. Tchuem Tchuente LA, Momo SC, Stothard JR, Rollinson D. Efficacy of praziquantel and reinfection patterns in single and mixed infection foci for intestinal and urogenital schistosomiasis in Cameroon. Acta Trop. 2013; 128(2):275-83.

5. Lamberton PHL, Kabatereine NB, Oguttu DW, Fenwick A, Webster JP. Sensitivity and specificity of multiple Kato-Katz thick smears and a circulating cathodic antigen test for Schistosoma mansoni diagnosis pre- and postrepeated-praziquantel treatment. PLoS Negl Trop Dis. 2014;8(9):e3139.

6. Katz N, Chaves A, Pellegrino JA. Simple device for quantitative stool thicksmear technique in schistosomiasis mansoni. Rev Inst Med Trop São Paulo. 1972;14(6):397-400.

7. Engels D, Sinzinkayo E, Gryseels B. Day-to-day egg count fluctuation in Schistosoma mansoni infection and its operational implications. Am J Trop Med Hyg. 1996;54(4):319-24.

8. Vennervald BJ, Ouma JH, Butterworth AE. Morbidity in schistosomiasis: assessment, mechanisms and control. Parasitol Today. 1998;14(10):385-90.

9. Utzinger J, Booth M, N'Goran EK, Múller I, Tanner M, Lengeler C. Relative contribution of day-to-day and intra-specimen variation in faecal egg counts of Schistosoma mansoni before and after treatment with praziquantel. Parasitology. 2001;122(5):537-44.

10. da Frota SM, Carneiro TR, Queiroz JA, Alencar LM, Heukelbach J, Bezerra FS. Combination of Kato-Katz faecal examinations and ELISA to improve accuracy of diagnosis of intestinal schistosomiasis in a low-endemic setting in Brazil. Acta Trop. 2011;120(Suppl. 1):S138-41.

11. Enk MJ, Lima AC, Massara CL, Coelho PM, Schall VTA. Combined strategy to improve the control of Schistosoma mansoni in areas of low prevalence in Brazil. Am J Trop Med Hyg. 2008;78(1):140-6.

12. Siqueira LM, Coelho PM, Oliveira AA, Massara CL, Carneiro NF, Lima AC, et al. Evaluation of two coproscopic techniques for the diagnosis of schistosomiasis in a low-transmission area in the state of Minas Gerais, Brazil. Mem Inst Oswaldo Cruz. 2011;106(7):844-50.

13. Colley DG, Binder S, Campbell C, King CH, Tchuem Tchuenté LA, N'Goran EK, et al. A five-country evaluation of a point-of-care circulating cathodic antigen urine assay for the prevalence of Schistosoma mansoni. Am J Trop Med Hyg. 2013;88(3):426-32.

14. van Dam G, Corstjens P, de Dood C, Coulibaly J, LoVerde P, Kariuki T. Diagnosis of active schistosome infections through antigen detection in urine: detection of single worm infections. Trop Med Int Health. 2013;18:11.

15. Ortu G, Ndayishimiye O, Clements M, Kayugi D, Campbell JCH, Lamine MS, et al. Countrywide reassessment of Schistosoma mansoni infection in Burundi using a urine-circulating cathodic antigen rapid test: informing the National Control Program. Am J Trop Med Hyg. 2017;96(3):664-73.

16. Haggag AA, Rabiee A, Abd Elaziz KM, Gabrielli AF, Abdel Hay R, Ramzy RM. Mapping of Schistosoma mansoni in the Nile Delta, Egypt: assessment of the prevalence by the circulating cathodic antigen urine assay. Acta Trop. 2017:167:9-17.

17. Amin MA, Elsaadig AM, Osman HA. Evaluation of cathodic antigen urine tests for diagnosis of Schistosoma mansoni infection in Sudan. Saudi J Med Medicial Sci. 2017;5(1):56-61.

18. Adriko M, Standley CJ, Tinkitina B, Tukahebwa EM, Fenwick A, Fleming FM, et al. Evaluation of circulating cathodic antigen (CCA) urine-cassette assay as a survey tool for Schistosoma mansoni in different transmission settings within Bugiri District, Uganda. Acta Trop. 2014;136:50-7.

19. Ochodo EA, Gopalakrishna G, Spek B, Reitsma JB, van Lieshout L, Polman K, et al. Circulating antigen tests and urine reagent strips for diagnosis of active schistosomiasis in endemic areas. In: the Cochrane collaboration, editor. Cochrane database of systematic reviews [internet]. Chichester, UK: John Wiley \& Sons, Ltd; 2015. Available from: http://doi.wiley.com/10.1002/ 14651858.CD009579.pub2

20. Danso-Appiah A, Minton J, Boamah D, Otchere J, Asmah RH, Rodgers M, et al. Accuracy of point-of-care testing for circulatory cathodic antigen in the detection of schistosome infection: systematic review and meta-analysis. Bull World Health Organ. 2016;94(7):522-33A.

21. Kittur N, Castleman JD, Campbell JCH, King CH, Colley DG. Comparison of Schistosoma mansoni prevalence and intensity of infection, as determined by the circulating cathodic antigen urine assay or by the Kato-Katz fecal assay: a systematic review. Am J Trop Med Hyg. 2016;94(3):605-10.

22. Colley DG, Andros TS, Campbell JCH. Schistosomiasis is more prevalent than previously thought: what does it mean for public health goals, policies, strategies, guidelines and intervention programs? Infect Poverty. 2017;6(1):63.

23. Koukounari A, Donnelly CA, Moustaki I, Tukahebwa EM, Kabatereine NB, Wilson S, et al. A latent Markov modelling approach to the evaluation of circulating cathodic antigen strips for schistosomiasis diagnosis pre- and 
post-praziquantel treatment in Uganda. PLoS Comput Biol. 2013;9(12): e1003402.

24. Shane HL, Verani JR, Abudho B, Montgomery SP, Blackstock AJ, Mwinzi PN, et al. Evaluation of urine CCA assays for detection of Schistosoma mansoni infection in western Kenya. PLoS Negl Trop Dis. 2011;5(1):e951.

25. Atlija M, Prada JM, Gutiérrez-Gil B, Rojo-Vázquez FA, Stear MJ, Arranz JJ, et al, Implementation of an extended ZINB model in the study of low levels of natural gastrointestinal nematode infections in adult sheep. BMC Vet Res. 2016;12:97.

26. Vlas SJD, Gryseels B, Oortmarssen GJV, Polderman AM, Habbema JDFA Model for variations in single and repeated egg counts in Schistosoma mansoni infections. Parasitology. 1992;104(3):451-60.

27. Engels D, Sinzinkayo E, de Vlas SJ, Gryseels B. Intraspecimen fecal egg count variation in Schistosoma mansoni infection. Am J Trop Med Hyg. 1997;57(5):571-7.

28. R Core Team. R: A language and Environment for statistical computing. Vienna, Austria: R Foundation for Statistical Computing; 2016. Available from: https://www.R-project.org/

29. Plummer M. JAGS: A program for analysis of Bayesian graphical models using Gibbs sampling. In: Proceedings of the 3rd International Workshop on Distributed Statistical Computing (DSC 2003). Kurt Hornik, Friedrich Leisch \& Achim Zeileis (eds). Vienna, Austria; March 20-22, 2003. ISSN 1609-395X. https://www.r-project.org/conferences/DSC-2003/Proceedings/Plummer.pdf.

30. Denwood MJ. Runjags: an R package providing interface utilities, model templates, parallel computing methods and additional distributions for MCMC models in JAGS. J Stat Softw. 2016;71(9):1-25.

31. WHO. Schistosomiasis. Progress report 2001-2011 and strategic plan 2012-2020. Geneva: World Health Organization; 2013.

32. Gryseels B, Mbaye A, De Vlas SJ, Stelma FF, Guisse F, Van Lieshout L, et al. Are poor responses to praziquantel for the treatment of Schistosoma mansoni infections in Senegal due to resistance? An overview of the evidence. Tropical Med Int Health. 2001;6(11):864-73.

33. Cioli D, Pica-Mattoccia L. Praziquantel. Parasitol Res. 2003;90(Suppl. 1):3-9.

34. Irvine MA, Njenga SM, Gunawardena S, Njeri Wamae C, Cano J, Brooker SJ, et al. Understanding the relationship between prevalence of microfilariae and antigenaemia using a model of lymphatic filariasis infection. Trans $R$ Soc Trop Med Hyg. 2016;110(2):118-24.

\section{Submit your next manuscript to BioMed Central and we will help you at every step:}

- We accept pre-submission inquiries

- Our selector tool helps you to find the most relevant journal

- We provide round the clock customer support

- Convenient online submission

- Thorough peer review

- Inclusion in PubMed and all major indexing services

- Maximum visibility for your research

Submit your manuscript at www.biomedcentral.com/submit

) Biomed Central 